\title{
Micro-algae come of age as a platform for recombinant protein production
}

\author{
Elizabeth Specht • Shigeki Miyake-Stoner • \\ Stephen Mayfield
}

Received: 31 March 2010/Accepted: 7 June 2010/Published online: 17 June 2010

(C) The Author(s) 2010. This article is published with open access at Springerlink.com

\begin{abstract}
A complete set of genetic tools is still being developed for the micro-alga Chlamydomonas reinhardtii. Yet even with this incomplete set, this photosynthetic single-celled plant has demonstrated significant promise as a platform for recombinant protein expression. In recent years, techniques have been developed that allow for robust expression of genes from both the nuclear and plastid genome. With these advances, many research groups have examined the pliability of this and other micro-algae as biological machines capable of producing recombinant peptides and proteins. This review describes recent successes in recombinant protein production in Chlamydomonas, including production of complex mammalian therapeutic proteins and monoclonal antibodies at levels sufficient for production at economic parity with existing production platforms. These advances have also shed light on the details of algal protein production at the molecular level, and provide insight into the next steps for optimizing micro-algae as a useful platform for the production of therapeutic and industrially relevant recombinant proteins.
\end{abstract}

E. Specht $\cdot$ S. Miyake-Stoner $\cdot$ S. Mayfield $(\bowtie)$

San Diego Center for Algae Biotechnology, Department of Biological Sciences, University of California, San Diego, 9500 Gilman Dr., La Jolla, CA 92093-0368, USA

e-mail: smayfield@ucsd.edu
Keywords Chlamydomonas · Micro-algae · Recombinant protein expression . Therapeutic protein production

\section{Introduction}

As genetically accessible photosynthetic organisms, algae are now recognized for their potential as a platform for recombinant protein expression where large scale and reduced material costs are important. The most-commonly used eukaryotic model alga, Chlamydomonas reinhardtii, has recently been shown to be able to fill this role, and this review will primarily discuss the technical and biological advances made in recombinant protein production in this alga. Algae have now come of age as a platform for recombinant protein expression.

All three genomes (chloroplast, mitochondrial, and nuclear) can be transformed in Chlamydomonas, and each has distinct transcriptional, translational, and post-translational properties that make them distinct. Each of these genomes has been fully sequenced, providing a wealth of information and a strong foundation for targeted manipulation (Maul et al. 2002; Popescu and Lee 2007; Merchant et al. 2007). Recent efforts have primarily focused on understanding and improving gene transformation, mRNA transcript accumulation, and protein accumulation of nuclear and chloroplast recombinant genes. 
Chlamydomonas is an excellent system for reasons beyond its genetic and metabolic malleability. This alga has a rapid doubling time (about $10 \mathrm{~h}$ ), it is easily scaled in homogenous culture as an aqueous microbe, it can be grown either photoautotrophically or with acetate as a reduced carbon source, and it has a controllable and rapid sexual cycle (about 2 weeks) with stable and viable haploids.

Advantages of algal protein production

Protein production in plants provides a number of advantages not found in other production platforms. First, a major advantage that all plant protein production systems have over cell culture systems (including bacteria, yeast, and mammalian cell culture) is the potential for significant reduction in cost. It is estimated that protein production in transgenic plants can be as much as four orders of magnitude less expensive than production in mammalian cell culture, on a per gram of unpurified protein basis (Dove 2002). Secondly, plant-produced proteins are not susceptible to viral or prion contamination that can harm humans, as is always a concern with animal cell culture (Chebolu and Daniell 2009). Third, as eukaryotes, algae and other plants possess the chaperones and cellular machinery required to fold complex human proteins that bacteria and yeast may not be able to process properly (Franklin and Mayfield 2004). Finally, many species of green algae are considered GRAS (generally regarded as safe) (Rosenberg et al. 2008), meaning that if the protein can be expressed in a bioavailable form, purification steps could potentially be eliminated altogether.

Algae possess a number of advantages over transgenic plant systems for the production of recombinant proteins. They can be grown in contained bioreactors, reducing the risk of contamination of the production system by airborne contaminants, and also protecting the environment from any potential flow of transgenes into the surrounding ecosystem. Growth in containment also greatly reduces the potential for loss of the crop due to predation or pathogen attack. Algae progress from initial transformation to large-scale protein production in a matter of weeks, compared to timescales on the order of months or years in higher plants such as corn or tobacco (Franklin and Mayfield 2004). As micro-algae are all a single cell type, there should also be less variation in recombinant protein accumulation, making downstream processing more uniform.

Production of recombinant proteins in chloroplasts also possesses several unique attributes. At present transgenic proteins can accumulate to much higher levels in the chloroplast than when expressed from the nuclear genome, mainly because plastids lack gene silencing mechanisms and other mechanisms that reduce recombinant protein production from nuclear encoded genes (Bock 2007). Chloroplasts can be transformed with multiple genes in a single event, due to the availability of multiple insertion sites, as well as an ability to process polycistronic transcripts, allowing an entire gene cassette to be regulated by a single promoter (Rymarquis et al. 2006; Bock 2007). Additionally, proteins produced within the chloroplast are not glycosylated (Franklin and Mayfield 2005), which can prove useful in many applications such as producing antibodies that are similar to native antibodies in their ability to recognize their antigen, but whose lack of glycosylation prevents them from recruiting killer cells (Tran et al. 2009). In fact, it is estimated that over two-thirds of the therapeutic human monoclonal antibodies in the testing pipeline do not require glycosylation for therapeutic function (Dove 2002).

\section{Genetic tools and techniques}

Transformation techniques

The plastid genome can be reliably transformed through homologous recombination using bombardment by DNA-coated gold or tungsten particles (Koop et al. 2007). Nuclear transformation in algae can also be achieved by biolistic bombardment, but the preferred methods are electroporation or agitation with glass beads using a cell-wall defective strain (Eichler-Stahlberg et al. 2009; Leon and Fernandez 2007). New transformation techniques using the Cre/ lox recombination system have been demonstrated to recombine in the nuclear genome of Chlamydomonas (Heitzer and Zschoernig 2007). Robust in vivo recombinant reporters, including GFPs (Fuhrmann et al. 1999; Franklin et al. 2002) and luciferases (Mayfield and Schultz 2004; Shao and Bock 2008), have been developed for tracking both nuclear and 
chloroplast gene expression. Techniques that have been employed previously on higher plants, such as transformation by Agrobacterium tumefaciens, have also been demonstrated to work with Chlamydomonas (Kumar et al. 2004). However, nuclear transformants still generally fail to accumulate recombinant proteins to the levels observed in plastids, most likely due to nuclear silencing mechanisms (see Nuclear Gene Silencing below).

\section{Codon optimization}

As with other expression systems, codon optimization has played a large role in the success of recombinant protein expression in both the chloroplast and nuclear genomes of Chlamydomonas (Heitzer et al. 2007). The nuclear genome and the plastid genome have highly divergent codon usage, with the chloroplast preferring an $\mathrm{A}$ or $\mathrm{T}$ in the wobble position while the nuclear genome prefers a $\mathrm{G}$ or $\mathrm{C}$ (Nakamura et al. 2000). Using GFP, early work showed that codon optimization to reflect the genome bias could increase transgene protein accumulation 5-fold in the nucleus (Fuhrmann et al. 1999) and up to 80-fold in the chloroplast (Franklin et al. 2002). Today, recombinant genes are universally codon optimized for improved protein expression in almost every system (Xia 2007; Puigbo et al. 2007, 2008).

\section{Chloroplast gene regulation}

Promoter and regulatory mRNA untranslated region (UTR) choices are both crucial factors in transgene expression levels. In chloroplasts, the most successful promoter to date in algae is the $p s b A$ promoter in combination with the $p s b A$ UTRs (Manuell et al. 2007; Surzycki et al. 2009). However, there are two caveats with using the $p s b A$ regulatory elements. First, they appear to be highly auto-attenuated; if any of the $p s b A$ gene product (D1 protein) is present, it will strongly decrease expression of any recombinant coding sequence under the control of its $5^{\prime}$-UTR. Secondly, since D1 is essential for the function of photosystem II, psbA knockouts are nonphotosynthetic. This would clearly negate the benefits of using a photosynthetic organism for protein production. There is evidence that reintroducing an attenuated $p s b A$ gene at a new locus elsewhere in the plastid genome can restore photosynthesis while only mildly reducing recombinant protein production (Manuell et al. 2007).

Other UTRs in use for transgene expression include those from the endogenous atpA, $r b c L$, and psbD genes (Fletcher et al. 2007; Hallmann 2007). These have been used with varying levels of success, though as with the $p s b A$ promoter, it is unclear why certain regulatory elements engender high expression levels with some genes but not others (Marin-Navarro et al. 2007). While endogenous promoters have been primarily used, other exogenously induced expression systems have been explored in the chloroplast. It has been demonstrated that inducible systems, such as the lac operon system from E. coli, can be engineered into the Chlamydomonas chloroplast (Kato et al. 2007), and more recently a riboswitch was shown to work to regulate translation in Chlamydomonas chloroplasts using a small molecule for induction (Croft et al. 2007), similar to their use in many other expression platforms (Suess 2005; Winkler and Breaker 2005).

While regulation of gene expression occurs at both the transcriptional and translational level in the nucleus, it appears that most regulation is posttranscriptional in the plastid (Marin-Navarro et al. 2007). However, many of the activators and suppressors of mRNA splicing and processing in the chloroplast are indeed encoded by nuclear genes (Boudreau et al. 2000; Somanchi et al. 2005; Raynaud et al. 2007; Schwarz et al. 2007; Loiselay et al. 2008). A multi-component copper-induced system has been designed as a switch for chloroplast protein expression. This system utilizes the nuclearencoded Nac 2 chloroplast protein necessary for stable accumulation of $p s b D$ RNA by acting on its 5 -regulatory region. By transforming a copper induced cytochrome c6 promoter fused to the Nac2 coding sequence into a Nac2 deficient strain, proteins encoded with the $p s b D$ regulatory region only accumulate in the presence of copper (Surzycki et al. 2007). Additionally, nuclear gene products are required for splicing of group $I$ introns in the chloroplast $23 \mathrm{~S}$ rRNA and $p s b A$ genes, and can potentially be used to regulate plastid gene expression (Li et al. 2002).

Post-transcriptional control in the chloroplast is mediated by both cis- and trans-acting-elements. The 5'-UTR of chimeric chloroplast mRNAs was shown to significantly impact recombinant protein production, 
while 3'-UTRs had little if any effect (Barnes et al. 2005). Cis-acting elements in $3^{\prime}$-UTRs include UGrepeats that are used in circadian protein regulation (Kiaulehn et al. 2007) and inverted repeat sequences that contribute to mRNA processing (GoldschmidtClermont et al. 2008). Recent work has identified a complex comprising an RNA stabilizing factor and a translational activator that appears to be specific to the D2 protein of photosystem II (Schwarz et al. 2007). Post-transcriptional control mechanisms can also have synergistic or antagonistic effects. Kasai et al. (2003) used the GUS reporter controlled by various endogenous $3^{\prime}$ - and $5^{\prime}$-UTRs to determine that there is an inverse correlation between protein accumulation and transcript stability, suggesting a feedback mechanism. Together all of these data suggest that regulation of mRNA translation has the greatest impact on recombinant protein accumulation, and is clearly an area where additional research seems likely to identify mechanisms for increased recombinant protein accumulation. For a comprehensive review of chloroplast translation regulation, see Marin-Navarro et al. 2007.

Nuclear gene regulation

In the nucleus, the most commonly used promoters are those from the HSP70A, psaD, and $r b c S 2$ genes (Schroda et al. 2002; Fischer and Rochaix 2001; Berthold et al. 2002). Chimeric promoters have also demonstrated high levels of transcription and expression (Schroda et al. 2000; Fischer and Rochaix 2001; $\mathrm{Wu}$ et al. 2008). Additionally, placing endogenous intronic sequences in transgenes has been shown to enhance expression regardless of orientation or position. The first intron from the endogenous rbcS2 gene has shown particular efficacy in increasing mRNA and protein accumulation and is now commonly used to enhance recombinant gene expression (Lumbreras et al. 1998).

In the nucleus, gene expression can be induced by a number of factors, including heat-shock or metal addition (Wu et al. 2008; Ferrante et al. 2008). The Nit1 promoter suppresses transcription in the presence of ammonia, but induces transcription when cells are grown in nitrate- or nitrite-containing media (Ohresser et al. 1997). More recently, iron-deficiency response elements (FeREs) in nuclear gene promoters have been characterized in Chlamydomonas (Fei and
Deng 2007). Signaling cascades triggered by photooxidative stress in the chloroplast can also activate transcription of specific nuclear genes, indicating that gene regulation in Chlamydomonas is not necessarily localized to the site of signal production (Fischer et al. 2007). More recently, signaling molecules such as $\mathrm{Mg}$-protoporphyrin and heme produced in the plastid have been shown to activate transcription of nuclear genes such as HSP70A through an interaction with the plastid response element (PRE) (von Gromoff et al. 2008). While these inducible systems provide great insight into gene regulatory strategies, there is still significant work to be done before robust production of recombinant proteins can be routinely achieved from nuclear genes in algae.

Nuclear gene silencing

Transgene silencing is a significant obstacle for recombinant protein expression in Chlamydomonas nuclear transformants, but recent work is helping to overcome this problem (Casas-Mollano et al. 2007, 2008a, b). Neupert et al. (2009) have developed strains with impaired transgene silencing by using UV mutagenesis and selection on media that permits higher antibiotic tolerance proportional to higher expression of the transgene product, to select strains with improved protein accumulation. However, their most impressive yields of exogenous protein accumulation are only $0.2 \%$ of total soluble protein (TSP), as compared to nearly $10 \%$ TSP obtained in plastids (Manuell et al. 2007). It has been postulated that gene silencing may be difficult to eliminate because it may have evolved as a protective measure against intracellular pathogens or viruses (Rosenberg et al. 2008; Neupert et al. 2009). It appears that combating gene silencing will be a major hurdle before recombinant proteins can be expressed at economically viable levels from nuclear transgenes in Chlamydomonas.

\section{Current successes in algal protein production}

Plastid transformation in higher plants has identified a few recombinant proteins that accumulate to very high levels, reported up to as high as $70 \%$ of total protein for some antibiotic proteins in tobacco leaves (Oey et al. 2009), but in general recombinant protein expression is highly variable. For an extensive review 
of vaccine production in plants, see Davoodi-Semiromi et al. (2009). However, very recent work in Chlamydomonas has demonstrated that fully bioactive proteins can indeed be produced to appreciable levels in green algae. A recent technique has been exploited to improve protein accumulation and stability by expression of cleavable fusions to highly expressed endogenous proteins (Muto et al. 2009), or to highly expressed recombinant proteins in the chloroplast (Rasala et al. 2010).

The first demonstration of mammalian protein expression in the chloroplast was of a large singlechain antibody (HSV8-lsc) directed against glycoprotein D of the herpes simplex virus (Mayfield et al. 2003). The protein was soluble, suggesting that it was correctly folded, and electrophoresis indicated the formation of the dimer by disulfide bond formation. This work was followed up by the expression of a single chain fragment variable antibody that accumulated to $0.54 \%$ TSP (Mayfield and Franklin 2005). More recently a full-length human IgG1 monoclonal antibody, directed against anthrax protective antigen 83 (83K7C), was expressed in the chloroplast of Chlamydomonas (Tran et al. 2009). Unlike the previously expressed lsc antibody, this antibody was assembled in the chloroplast from separately expressed light chain and heavy chain proteins, and it could be purified at $100 \mu \mathrm{g}$ per $1 \mathrm{~g}$ dry algal biomass, and was found to have binding activity identical to that of the same antibody expressed in a traditional mammalian cell culture system (Tran et al. 2009).

A host of non-antibody recombinant proteins have been expressed in the chloroplast for therapeutic purposes. The human metallothionein- 2 gene product, which is considered to have anti-radiation function, was expressed and demonstrated to improve the survivorship of transgenic algae compared to wild type algae (Zhang et al. 2006). The human tumor necrosis factor-related apoptosis-inducing ligand (TRAIL) protein, known to induce apoptosis in virus-infected and tumor cells, accumulated in the chloroplast at $0.43-0.67 \%$ TSP based on densitometric analysis of Western blot (Yang et al. 2006). The expression of human glutamic acid decarboxylase (hGAD65) was also achieved in the chloroplast of Chlamydomonas (Wang et al. 2008). This protein is an important autoantigenic marker in type I diabetes, and the algae-derived protein remained immunologically active, accumulating at $0.25-0.3 \%$ TSP. Human erythropoietin (Epo), used in the treatment of anemia, was fused to an export sequence and expressed from the nuclear genome (EichlerStahlberg et al. 2009). Protein was detected in and isolated from the culture medium, although with uncharacterized post-translational modification closely matching the mass of endogenous human Epo. Also, biologically active bovine mammary-associated serum amyloid (M-SAA) was expressed in the chloroplast (Manuell et al. 2007). Notably, the accumulation of this soluble protein was above $5 \%$ TSP as quantified by Western blot, with levels of expression determined to be more than twice that amount using ELISA quantification.

A fusion protein containing the foot-and-mouth disease virus VP1 gene and the cholera toxin B subunit (CTBVP1) was produced in the chloroplast as well (Sun et al. 2003). The protein was reported to accumulate to $3 \%$ total protein by ELISA quantification, and retained GM1-ganglioside binding activity and antigenicity. Another viral protein, classical swine fever virus E2 structural protein, was expressed in the chloroplast (He et al. 2007). ELISA quantification indicated the accumulation of the $\mathrm{E} 2$ protein to $1.5-2 \%$ TSP, and retained immunological activity. Similarly, along with a list of other recombinant proteins, the white spot syndrome virus protein 28 (VP28) was expressed in the chloroplast (Surzycki et al. 2009). VP28 was reported to accumulate to a striking $10.5 \%$ TSP, although no data was presented to show how this level of expression was determined. Recently, the D2 fibronectin-binding domain from a Staphylococcus aureus protein was fused to the B subunit of cholera toxin, and expressed in the chloroplast (Dreesen et al. 2010). The transgenic algae were fed to mice, and induced resistance against lethal doses of $S$. aureus, presumably by eliciting a systemic antigenic response to the $S$. aureus peptide. This is the first demonstration of the functional possibility of orally delivered vaccines from algal production.

More recently, Rasala et al. (2010) attempted the expression of a set of seven recombinant proteins in the chloroplast and were met with very good success (four out of the seven genes expressed at economically viable levels). This work demonstrates that recombinant protein expression in algal chloroplasts is on par with any other expression platform, and 
shows that expression of complex mammalian proteins is as likely to be achieved in algae as it is in any eukaryotic system. These data are summarized in Table 1.

\section{Potential future applications}

Oral vaccines

Traditional vaccines are normally produced from an attenuated or killed form of the pathogenic organism itself. An alternative approach is to produce a pathogen antigen as a recombinant protein, and this is now used for some select vaccines like the hepatitis A vaccine (Powdrill and Johnston 1991). As algae contain very sophisticated protein folding machinery that bacteria and other prokaryotes lack, algae can be used to produce complex eukaryotic proteins that cannot be easily produced on large scale in bacterial culture without costly denaturation and refolding steps. Algae are also ideal for producing vaccines against pathogens which exhibit little or no glycosylation, such as those from the parasites plasmodium (Gowda and Davidson 1999).

Algae are also especially suited for the production of oral vaccines on a large scale due to their GRAS status. Once the process of oral delivery of a vaccine protein is refined, algae could produce inexpensive oral vaccines, making vaccination an accessible form of disease management for a whole host of thirdworld diseases. Furthermore, the oral delivery method and the option to store doses at ambient temperature would allow vaccines to be transported and administered to remote populations without the need for expensive refrigeration or highly trained medical personnel (Chebolu and Daniell 2009).

Evidence for the feasibility of plastid-produced vaccines has been provided by several groups who have produced antigens that elicit similar immune responses as the actual pathogen when injected in standard vaccine adjuvants (Tregoning et al. 2003; Koya et al. 2005; Molina and Shoenfeld 2005; Chebolu and Daniell 2009). Furthermore, fusions of antigens with the cholera toxin B subunit show promise for eliciting immune response from mucosal delivery alone, as the cholera toxin B subunit allows a fused protein to penetrate the intestinal lining (Sun et al. 2003; Harakuni et al. 2005).
Discovery of novel bioactive molecules

Screening of diverse algal species for novel bioactive compounds has become a field of intense interest lately, as algae are a rich source of secondary metabolites that often have industrial or nutritional implications. A wide range of functional molecules produced by algae - everything from antioxidants to pigments used in laboratory analytical techniqueshave been isolated (Plaza et al. 2009). High-throughput screening methods comprising pressurized liquid extraction; functional characterization of antioxidant or antimicrobial activity; and chemical characterization by HPLC and GC-MS have been optimized to streamline the screening process (Plaza et al. 2010).

\section{Protein secretion}

Figure 1 illustrates the basic structure of Chlamydomonas reinhardtii, as well as the cellular locations of recombinant protein accumulation depending on the gene construct and transformation method. Plastid transformation results in accumulation of the transgene product in the single large chloroplast. Nuclear gene products accumulate in the cytosol by default, but nuclear transformation with appropriate signal sequences allows for targeting to the endoplasmic reticulum and Golgi for export (Griesbeck et al. 2006) or for localization to the cell membrane, which may be sufficient for antigenic recognition in the case of an oral vaccine (Eichler-Stahlberg et al. 2009).

\section{Other algal transformation efforts}

Genetic manipulation of algae is no longer limited to Chlamydomonas reinhardtii. Recent successes in generating transgenic algae are growing in number. Exogenous genes have been expressed in the unicellular charophyte alga, Closterium peracerosum-strigosum-littorale complex (Abe et al. 2008). The nuclear genome of volvocine alga Gonium pectorale has been stably transformed (Lerche and Hallmann 2009), as has the chlorophyceae Haematococcus pluvialis (Kathiresan and Sarada 2009), by co-cultivation with Agrobacterium (Kathiresan et al. 2009). Some successes have been made by transient transformation of marine chlorarachniophyte Lotharella amoebiformis (Hirakawa et al. 2008), chlorophyta alga Ulva pertusa 


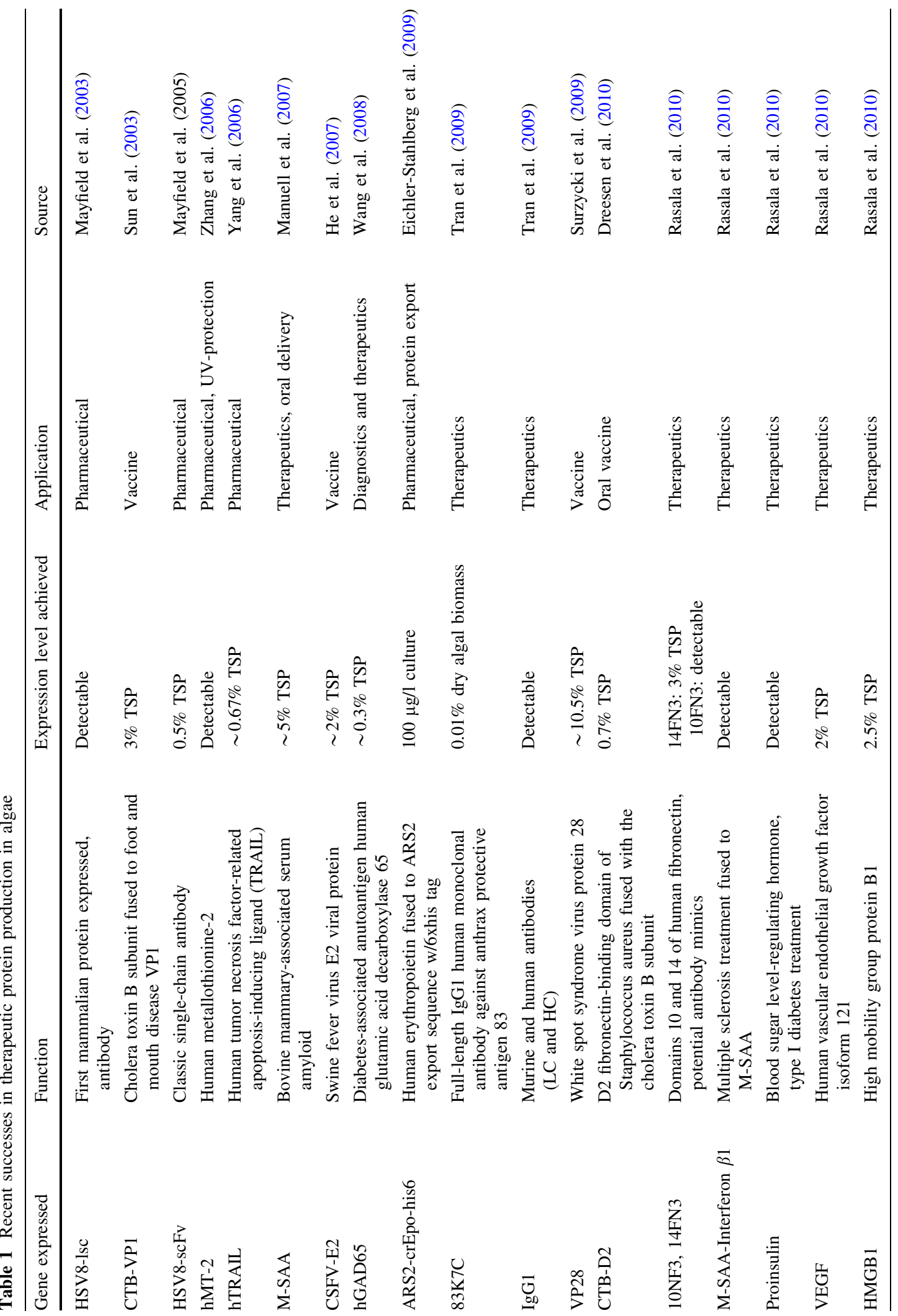




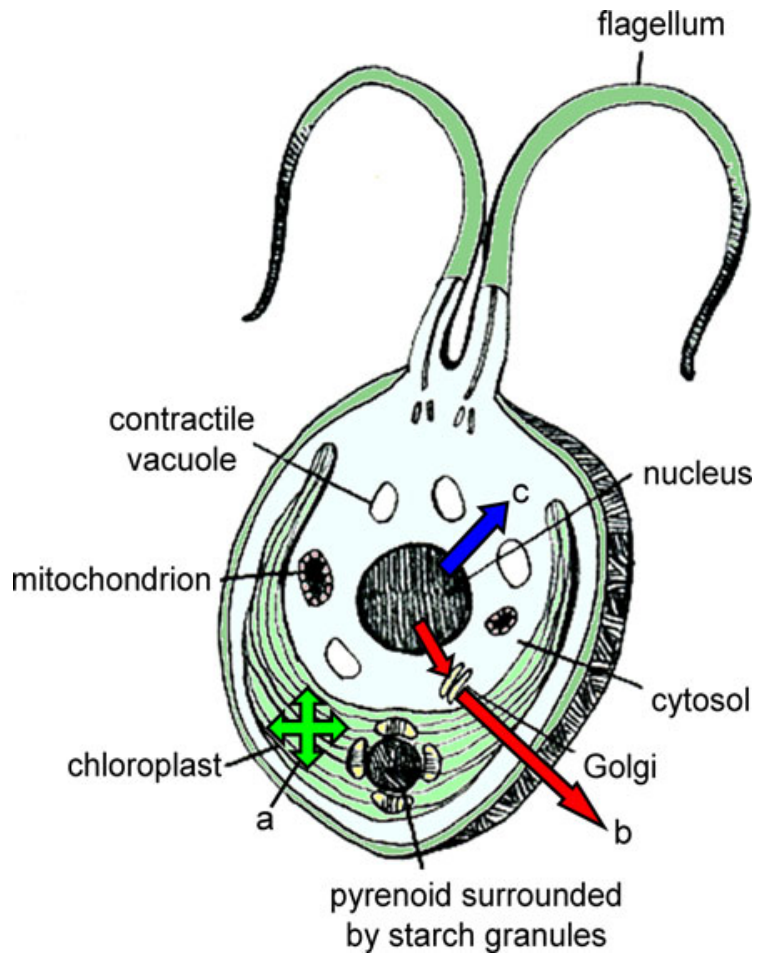

Fig. 1 Chlamydomonas reinhardtii as a versatile recombinant protein production platform. Protein expressed from the chloroplast genome is accumulated inside the single large chloroplast $(a)$. The reducing environment of the chloroplast allows for proper folding of heavily disulfide bonded proteins, which is not easily accomplished in bacterial production platforms. Protein expressed from the nuclear genome accumulates in the cytosol $(b)$, unless it is given an export signal sequence. In this case, it is sent to the endoplasmic reticulum for translocation and processing and then moves to the Golgi apparatus for packaging and export to the extracellular media $(c)$

(Kakinuma et al. 2009), and red alga Cyanidioschyzon merolae (Ohnuma et al. 2008). Methods have also been improved for previously transformed alga, such as Dunaliella salina (Feng et al. 2009), and cyanobacterial genetics have also been extensively explored, but will not be discussed in this review.

\section{Future challenges}

A significant obstacle for algal protein expression systems is the lack of production systems optimized for large-scale growth and harvesting of algae under photoautotrophic conditions. The main limitations in photobioreactor size arise from inhibited gas exchange and light penetration in large cultures, especially at the high cell densities required to keep costs low (Ugwu et al. 2008). An alternative approach, which has been discussed extensively by Chen and Chen (2006), is to grow algae heterotrophically in conventional fermentation bioreactors. This is certainly an economically viable option for high-value products such as therapeutics or enzymes, and indeed is currently used in much of the microalgae products industry (Chen and Chen 2006) with yields reported as high as $83 \mathrm{~g}$ dry weight per liter of culture for some species (de Swaaf et al. 2003). Maximum yields for Chlamydomonas reinhardtii are around $1.5 \mathrm{~g}$ dry biomass per liter when grown in continuous flow on acetate media (Chen and Johns 1996).

The scale and cost requirements for algal biofuels will likely necessitate photoautotrophic open cultivation systems. These systems are being improved, as are processes for efficiently harvesting the algae. For example, new methods have been optimized for harvesting algae using microbes capable of flocculating $90 \%$ of the algal mass with no deleterious effect on algal viability (Lee et al. 2009). More efficient methods and systems for large-scale growth are critical if algal-derived biofuels are to become a reality. If these issues can be resolved, algae represent a far superior source of biofuel than terrestrial plants. It is estimated algae can produce up to ten times as much oil per acre than any current terrestrial crop (Cooney et al. 2009). For a detailed recent review on the potential of algal biofuels, see Mata et al. 2010.

\section{Conclusion}

Several decades of work in Chlamydomonas has elucidated a better understanding of the transcriptional and translational machinery and regulation of the cell, ultimately generating improved methods for transgenic expression of recombinant proteins in algae. However, only in the past few years have these advances been used to successfully express large, fully bioactive, therapeutically relevant proteins at a level sufficient for economically viable large-scale production. Extensive research on optimal transformation constructs and gene optimization have greatly increased yields of recombinant protein, though further work is still needed to address nuclear gene 
silencing, plastid auto-attenuation, and to optimize reactor design for large-scale use. Algae have proven their utility and tractability as a production system for therapeutic or industrial proteins and peptides, and algae now seem poised to become the "green" alternative to current mammalian, yeast, or bacterial recombinant protein production systems.

Acknowledgements This work was supported by a grant from the National Institute of Allergy and Infectious Diseases to SPM (AI059614). ES is supported by a National Science Foundation pre-doctoral fellowship.

Open Access This article is distributed under the terms of the Creative Commons Attribution Noncommercial License which permits any noncommercial use, distribution, and reproduction in any medium, provided the original author(s) and source are credited.

\section{References}

Abe J, Hiwatashi Y, Ito M et al (2008) Expression of exogenous genes under the control of endogenous HSP70 and $\mathrm{CAB}$ promoters in the Closterium peracerosum-strigosum-littorale complex. Plant Cell Physiol 49:625-632

Barnes D, Franklin S, Schultz J et al (2005) Contribution of $5^{\prime}$ - and $3^{\prime}$-untranslated regions of plastid mRNAs to the expression of Chlamydomonas reinhardtii chloroplast genes. Mol Genet Genomics 274:625-636

Berthold P, Schmitt R, Mages W (2002) An engineered Streptomyces hygroscopicus aph 7" gene mediates dominant resistance against hygromycin B in Chlamydomonas reinhardtii. Protist 153:401-412

Bock R (2007) Plastid biotechnology: prospects for herbicide and insect resistance, metabolic engineering and molecular farming. Curr Opin Biotechnol 18:100-106

Boudreau E, Nickelsen J, Lemaire SD et al (2000) The Nac2 gene of Chlamydomonas encodes a chloroplast TPR-like protein involved in psbD mRNA stability. Eur Mol Biol Organ J 19:3366-3376

Casas-Mollano JA, van Dijk K, Eisenhart J et al (2007) SET3p monomethylates histone $\mathrm{H} 3$ on lysine 9 and is required for the silencing of tandemly repeated transgenes in Chlamydomonas. Nucleic Acids Res 35:939-950

Casas-Mollano JA, Jeong B-r, Xu J et al (2008a) The MUT9p kinase phosphorylates histone $\mathrm{H} 3$ threonine 3 and is necessary for heritable epigenetic silencing in Chlamydomonas. Proc Natl Acad Sci 105:6486-6491

Casas-Mollano JA, Rohr J, Kim E-J et al (2008b) Diversification of the core RNA interference machinery in Chlamydomonas reinhardtii and the role of DCL1 in transposon silencing. Genetics 179:69-81

Chebolu S, Daniell H (2009) Chloroplast-derived vaccine antigens and biopharmaceuticals: expression, folding, assembly and functionality. Plant-Produced Microbial Vaccines, pp 33-54

Chen G-Q, Chen F (2006) Growing phototrophic cells without light. Biotechnol Lett 28:607-616
Chen F, Johns MR (1996) Heterotrophic growth of Chlamydomonas reinhardtii on acetate in chemostat culture. Process Biochem 31:601-604

Cooney M, Young G, Nagle N (2009) Extraction of bio-oils from microalgae. Sep Purif Rev 38:291-325

Croft MT, Moulin M, Webb ME et al (2007) Thiamine biosynthesis in algae is regulated by riboswitches. Proc Natl Acad Sci 104:20770-20775

Davoodi-Semiromi A, Samson N, Daniell H (2009) The green vaccine: a global strategy to combat infectious and autoimmune diseases. Hum Vaccin 5:488-493

de Swaaf ME, Pronk JT, Sijtsma L (2003) Fed-batch cultivation of the docosahexacaoic-acid-producing marine alga Crypthecodinium colunii on ethanol. Appl Microbiol Biotechnol 61:40-43

Dove A (2002) Uncorking the biomanufacturing bottleneck. Nat Biotechnol 20:777-779

Dreesen IAJ, Charpin-El Hamri G, Fussenegger M (2010) Heat-stable oral alga-based vaccine protects mice from Staphylococcus aureus infection. J Biotechnol 145:273280

Eichler-Stahlberg A, Weisheit W, Ruecker O et al (2009) Strategies to facilitate transgene expression in Chlamydomonas reinhardtii. Planta 229:873-883

Fei XW, Deng XD (2007) A novel Fe deficiency-responsive element (FeRE) regulates the expression of atx 1 in Chlamydomonas reinharditii. Plant Cell Physiol 48:1496-1503

Feng S, Xue L, Liu H et al (2009) Improvement of efficiency of genetic transformation for Dunaliella salina by glass beads method. Mol Biol Rep 36:1433-1439

Ferrante P, Catalanotti C, Bonente G et al (2008) An optimized, chemically regulated gene expression system for Chlamydomonas. PLoS ONE 3(9): 3200

Fischer N, Rochaix JD (2001) The flanking regions of PsaD drive efficient gene expression in the nucleus of the green alga Chlamydomonas reinhardtii. Mol Genet Genomics 265:888-894

Fischer BB, Krieger-Liszkay A, Hideg E et al (2007) Role of singlet oxygen in chloroplast to nucleus retrograde signaling in Chlamydomonas reinhardtii. FEBS Lett 581:5555-5560

Fletcher SP, Muto M, Mayfield SP (2007) Optimization of recombinant protein expression in the chloroplasts of green algae. In: Leon R, Galvan A, Fernandez E (eds) Advances in experimental medicine and biology, vol 616: transgenic microalgae as green cell factories. Landes Bioscience and Springer Science Business Media LLC, New York, NY, pp 90-98

Franklin SE, Mayfield SP (2004) Prospects for molecular farming in the green alga Chlamydomonas reinhardtii. Curr Opin Plant Biol 7:159-165

Franklin SE, Mayfield SP (2005) Recent developments in the production of human therapeutic proteins in eukaryotic algae. Expert Opin Biol Ther 5:225-235

Franklin S, Ngo B, Efuet E et al (2002) Development of a GFP reporter gene for Chlamydomonas reinhardtii chloroplast. Plant J 30:733-744

Fuhrmann M, Oertel W, Hegemann P (1999) A synthetic gene coding for the green fluorescent protein (GFP) is a versatile reporter in Chlamydomonas reinhardtii. Plant $\mathrm{J}$ 19:353-361 
Goldschmidt-Clermont M, Rahire M, Rochaix JD (2008) Redundant cis-acting determinants of $3^{\prime}$ processing and RNA stability in the chloroplast rbcL mRNA of Chlamydomonas. Plant J 53:566-577

Gowda DC, Davidson EA (1999) Protein glycosylation in the malaria parasite. Parasitol Today 15:147-152

Griesbeck C, Kobl I, Heitzer M (2006) Chlamydomonas reinhardtii. Mol Biotechnol 34:213-223

Hallmann A (2007) Algal transgenics and biotechnology. Transgenic Plant J 1:81-98

Harakuni T, Sugawa H, Komesu A et al (2005) Heteropentameric cholera toxin B subunit chimeric molecules genetically fused to a vaccine antigen induce systemic and mucosal immune responses: a potential new strategy to target recombinant vaccine antigens to mucosal immune systems. Infect Immun 73:5654-5665

He D-M, Qian K-X, Shen G-F et al (2007) Recombination and expression of classical swine fever virus (CSFV) structural protein E2 gene in Chlamydomonas reinhardtii chloroplasts. Colloids Surf B Biointerfaces 55:26-30

Heitzer M, Zschoernig B (2007) Construction of modular tandem expression vectors for the green alga Chlamydomonas reinhardtii using the Cre/lox-system. Biotechniques 43:324-328

Heitzer M, Eckert A, Fuhrmann M et al (2007) Influence of codon bias on the expression of foreign genes in microalgae. Transgenic Microalgae as Green Cell Factories, pp 46-53

Hirakawa Y, Kofuji R, Ishida K-i (2008) Transient transformation of a chlorarachniophyte alga, Lotharella amoebiformis (Chlorarachniophyceae), with uidA and egfp reporter genes. J Phycol 44:814-820

Kakinuma M, Ikeda M, Coury D et al (2009) Isolation and characterization of the rbcS genes from a sterile mutant of Ulva pertusa (Ulvales, Chlorophyta) and transient gene expression using the rbcS gene promoter. Fish Sci 75:1015-1028

Kasai S, Yoshimura S, Ishikura K et al (2003) Effect of coding regions on chloroplast gene expression in Chlamydomonas reinhardtii. J Biosci Bioeng 95:276-282

Kathiresan S, Sarada R (2009) Towards genetic improvement of commercially important microalga Haematococcus pluvialis for biotech applications. J Appl Phycol 21:553-558

Kathiresan S, Chandrashekar A, Ravishankar GA et al (2009) Agrobacterium-mediated transformation in the green alga Haematococcus pluvialis (Chlorophyceae, Volvocales). J Phycol 45:642-649

Kato K, Marui T, Kasai S et al (2007) Artificial control of transgene expression in Chlamydomonas reinhardtii chloroplast using the lac regulation system from Escherichia coli. J Biosci Bioeng 104:207-213

Kiaulehn S, Voytsekh O, Fuhrmann M et al (2007) The presence of UG-repeat sequences in the $3^{\prime}$-UTRs of reporter luciferase mRNAs mediates circadian expression and can determine acrophase in Chlamydomonas reinhardtii. J Biol Rhythms 22:275-277

Koop H-U, Herz S, Golds T (2007) The genetic transformation of plastids. In: Bock Ralph (ed) Topics in current genetics, vol 19: cell and molecular biology of plastids. Springer, Berlin/Heidelberg, pp 457-510

Koya V, Moayeri M, Leppla SH et al (2005) Plant-based vaccine: mice immunized with chloroplast-derived anthrax protective antigen survive anthrax lethal toxin challenge. Infect Immunol 73:8266-8274

Kumar SV, Misquitta RW, Reddy VS et al (2004) Genetic transformation of the green alga Chlamydomonas reinhardtii by Agrobacterium tumefaciens. Plant Sci 166:731-738

Lee AK, Lewis DM, Ashman PJ (2009) Microbial flocculation, a potentially low-cost harvesting technique for marine microalgae for the production of biodiesel. J Appl Phycol 21:559-567

Leon R, Fernandez E (2007) Nuclear transformation of eukaryotic microalgae-historical overview, achievements and problems. In: Leon R, Fernandez E (eds) Transgenic microalgae as green cell factories, vol 616. Landes Bioscience and Springer Science Business Media LLC, New York, pp 1-11

Lerche K, Hallmann A (2009) Stable nuclear transformation of Gonium pectorale. BMC Biotechnol 9:64

Li F, Holloway SP, Lee J et al (2002) Nuclear genes that promote splicing of group I introns in the chloroplast $23 \mathrm{~S}$ rRNA and psbA genes in Chlamydomonas reinhardtii. Plant J 32:467-480

Loiselay C, Gumpel NJ, Girard-Bascou J et al (2008) Molecular identification and function of cis- and trans-acting determinants for petA transcript stability in Chlamydomonas reinhardtii chloroplasts. Mol Cell Biol 28:5529-5542

Lumbreras V, Stevens DR, Purton S (1998) Efficient foreign gene expression in Chlamydomonas reinhardtii mediated by an endogenous intron. Plant J 14:441-447

Manuell AL, Beligni MV, Elder JH et al (2007) Robust expression of a bioactive mammalian protein in Chlamydomonas chloroplast. Plant Biotechnol J 5:402-412

Marin-Navarro J, Manuell AL, Wu J et al (2007) Chloroplast translation regulation. Photosynth Res 94:359-374

Mata TM, Martins AA, Caetano NS (2010) Microalgae for biodiesel production and other applications: a review. Renew Sustain Energy Rev 14:217-232

Maul JE, Lilly JW, Cui L et al (2002) The Chlamydomonas reinhardtii plastid chromosome: islands of genes in a sea of repeats. Plant Cell 14:2659-2679

Mayfield SP, Franklin SE (2005) Expression of human antibodies in eukaryotic micro-algae. Vaccine 23:18281832

Mayfield S, Schultz J (2004) Development of a luciferase reporter gene, luxCt, for Chlamydomonas reinhardtii chloroplast. Plant J 37:449-458

Mayfield SP, Franklin SE, Lerner RA (2003) Expression and assembly of a fully active antibody in algae. Proc Natl Acad Sci 100:438-442

Merchant SS, Prochnik SE, Vallon O et al (2007) The Chlamydomonas genome reveals the evolution of key animal and plant functions. Science 318:245-250

Molina V, Shoenfeld Y (2005) Infection, vaccines and other environmental triggers of autoimmunity. Autoimmunity 38:235-245

Muto M, Henry Ryan E, Mayfield Stephen P (2009) Accumulation and processing of a recombinant protein designed as a cleavable fusion to the endogenous Rubisco LSU protein in Chlamydomonas chloroplast. BMC Biotechnol 9:26

Nakamura Y, Gojobori T, Ikemura T (2000) Codon usage tabulated from international dna sequence databases: status for the year 2000. Nucleic Acids Res 28:292 
Neupert J, Karcher D, Bock R (2009) Generation of Chlamydomonas strains that efficiently express nuclear transgenes. Plant J 57:1140-1150

Oey M, Lohse M, Kreikemeyer B et al (2009) Exhaustion of the chloroplast protein synthesis capacity by massive expression of a highly stable protein antibiotic. Plant $\mathbf{J}$ 57:436-445

Ohnuma M, Yokoyama T, Inouye T et al (2008) Polyethylene glycol (PEG)-mediated transient gene expression in a red alga, Cyanidioschyzon merolae 10D. Plant Cell Physiol 49:117-120

Ohresser M, Matagne RF, Loppes R (1997) Expression of the arylsulphatase reporter gene under the control of the nit1 promoter in Chlamydomonas reinhardtii. Curr Genet 31:264-271

Plaza M, Herrero M, Cifuentes A et al (2009) Innovative natural functional ingredients from microalgae. J Agric Food Chem 57:7159-7170

Plaza M, Santoyo S, Jaime L et al (2010) Screening for bioactive compounds from algae. J Pharm Biomed Anal 51:450-455

Popescu CE, Lee RW (2007) Mitochondrial genome sequence evolution in Chlamydomonas. Genetics 175:819-826

Powdrill TF, Johnston JM (1991) Immunologic priming with recombinant hepatitis A virus capsid proteins produced in Escherichia coli. J Virol 65:2686-2690

Puigbo P, Guzman E, Romeu A et al (2007) OPTIMIZER: a web server for optimizing the codon usage of DNA sequences. Nucleic Acids Res 35:W126-W131

Puigbo P, Bravo I, Garcia-Vallve S (2008) E-CAI: a novel server to estimate an expected value of codon adaptation index (eCAI). BMC Bioinformatics 9:65

Rasala BA, Muto M, Lee PA et al (2010) Production of therapeutic proteins in algae, analysis of expression of seven human proteins in the chloroplast of Chlamydomonas reinhardtii. Plant Biotechnol J 8:1-15

Raynaud C, Loiselay C, Wostrikoff K et al (2007) Evidence for regulatory function of nucleus-encoded factors on mRNA stabilization and translation in the chloroplast. Proc Natl Acad Sci 104:9093-9098

Rosenberg JN, Oyler GA, Wilkinson L et al (2008) A green light for engineered algae: redirecting metabolism to fuel a biotechnology revolution. Curr Opin Biotechnol 19:430-436

Rymarquis LA, Higgs DC, Stern DB (2006) Nuclear suppressors define three factors that participate in both $5^{\prime}$ and $3^{\prime}$ end processing of mRNAs in Chlamydomonas chloroplasts. Plant J 46:448-461

Schroda M, Blcker D et al (2000) The HSP70A promoter as a tool for the improved expression of transgenes in Chlamydomonas. Plant J 21:121-131

Schroda M, Beck CF, Vallon O (2002) Sequence elements within an HSP70 promoter counteract transcriptional transgene silencing in Chlamydomonas. Plant J 31:445455

Schwarz C, Elles I, Kortmann J et al (2007) Synthesis of the D2 protein of photosystem II in Chlamydomonas is controlled by a high molecular mass complex containing the RNA stabilization factor Nac2 and the translational activator RBP40. Plant Cell 19:3627-3639

Shao N, Bock R (2008) A codon-optimized luciferase from Gaussia princeps facilitates the in vivo monitoring of gene expression in the model alga Chlamydomonas reinhardtii. Curr Genet 53:381-388

Somanchi A, Barnes D, Mayfield SP (2005) A nuclear gene of Chlamydomonas reinhardtii, Tbal, encodes a putative oxidoreductase required for translation of the chloroplast psbA mRNA. Plant J 42:341-352

Suess B (2005) Engineered riboswitches control gene expression by small molecules. Biochem Soc Trans 33:474-476

Sun M, Qian KX, Su N et al (2003) Foot-and-mouth disease virus VP1 protein fused with cholera toxin B subunit expressed in Chlamydomonas reinhardtii chloroplast. Biotechnol Lett 25:1087-1092

Surzycki R, Cournac L, Peltier G et al (2007) Potential for hydrogen production with inducible chloroplast gene expression in Chlamydomonas. Proc Natl Acad Sci 104:17548-17553

Surzycki R, Greenham K, Kitayama K et al (2009) Factors effecting expression of vaccines in microalgae. Biologicals 37:133-138

Tran M, Zhou B, Pettersson PL et al (2009) Synthesis and assembly of a full-length human monoclonal antibody in algal chloroplasts. Biotechnol Bioeng 104:663-673

Tregoning JS, Nixon P, Kuroda $\mathrm{H}$ et al (2003) Expression of tetanus toxin Fragment $\mathrm{C}$ in tobacco chloroplasts. Nucleic Acids Res 31:1174-1179

Ugwu CU, Aoyagi H, Uchiyama H (2008) Photobioreactors for mass cultivation of algae. Bioresour Technol 99:40214028

von Gromoff ED, Alawady A, Meinecke L et al (2008) Heme, a plastid-derived regulator of nuclear gene expression in Chlamydomonas. Plant Cell 20:552-567

Wang XF, Brandsma M, Tremblay R et al (2008) A novel expression platform for the production of diabetes-associated autoantigen human glutamic acid decarboxylase (hGAD65). BMC Biotechnol 8:87

Winkler WC, Breaker RR (2005) Regulation of bacterial gene expression by riboswitches. Annu Rev Microbiol 59:487517

Wu JX, Hu ZL, Wang CG et al (2008) Efficient expression of green fluorescent protein (GFP) mediated by a chimeric promoter in Chlamydomonas reinhardtii. Chin J Oceanol Limnol 26:242-247

Xia X (2007) An improved implementation of codon adaptation index. Evol Bioinform Online 3:53-58

Yang Z, Li y, Chen F et al (2006) Expression of human soluble TRAIL in Chlamydomonas reinhardtii chloroplast. Chin Sci Bull 51:1703-1709

Zhang Y-K, Shen G-F, Ru B-G (2006) Survival of human metallothionein-2 transplastomic Chlamydomonas reinhardtii to ultraviolet B exposure. Acta Biochim Biophys Sin 38:187-193 